\title{
AN IMPROVED 3D FACE SYNTHESIS BASED ON MORPHABLE MODEL
}

\author{
Chengzhang Wang, Baocai Yin, Qin Shi, Yanfeng Sun \\ Beijing Municipal Multimedia and Intelligent Software Key Lab, Beijing University of \\ Technology, Beijing 100022, China
}

Abstract: A novel model matching method based on improved genetic algorithm is presented in this paper to improve efficiency of matching process for $3 \mathrm{D}$ face synthesis. New method is independent from initial values and more robust than stochastic gradient descent method. Improved genetic algorithm has strong global searching ability. Crossover and mutation probability are regulated during optimization process to improve precision and convergence speed of the algorithm. Experimental results show our new model matching method has good performance on 3D face synthesis.

Key words: Morphable model, 3D face synthesis, Genetic Algorithm

\section{INTRODUCTION}

Modeling human faces has challenged researchers in computer graphics since its beginning. Since the pioneering work of Parke [1], various methods have been reported for modeling the shape and texture of faces and for animating them $[2,3]$. In past several years, a novel face modeling method, the morphable model is proposed by T. Vetter [4]. Matching the model to given facial images, even a single image, realistic 3D face model of the person could be synthesized automatically by combination of prototypic faces. The method based on the morphable model is automatic and gives light on the difficult problems of facial research, illumination and poses evaluation. Stochastic gradient descent method is generally used to implement model matching process presently [4]. However, this method depends too much on initial values and to be time consuming. Convergence 
of the method is hardly well either [5]. Novel model matching method need to be studied to improve efficiency of the morphable model.

We will introduce the morphable model in following section. Model matching method based on improved genetic algorithm will be presented in section 3. In section 4, 3D face synthesis results are presented using the proposed method. Conclusions are presented in section 5.

\section{MORPHABLE MODEL}

3D face synthesis system based on the morpable model is composed of two components. One is model construction. The other is model matching process. To construct the morphable model, prototypic 3D faces are acquired firstly by CyberWare 3D Face Scanner [6]. After computing correspondence between the prototypes, every prototypic $3 \mathrm{D}$ face can be represented by a shape vector and a texture vector. Technique of PCA (principal component analysis) is used to give the final morphable model format [4]:

$$
S_{\bmod e l}=\bar{S}+\sum_{i=1}^{m} \alpha_{i} s_{i} \quad T_{\bmod e l}=\bar{T}+\sum_{i=1}^{m} \beta_{i} t_{i}
$$

Matching the morphable model to given facial images is the process of face synthesis. By model matching process, reconstructed 3D face is obtained by combination operation. Object function is defined as follows [4]:

$$
E=\sum_{(x, y)}\left[I_{\text {input }}(x, y)-I_{\bmod e l}(x, y)\right]^{2}
$$

\section{MODEL MATCHING BASED ON IMPROVED GENETIC ALGORITHM}

Genetic algorithm is one of the modern optimization methods [7]. Improved genetic algorithm is proposed to solve model matching process.

\subsection{Genetic Operator}

Floating encoding and roulette selection method are adopted in this work. Evaluation function is designed by the formula: $F(X)=C_{\max }-E$, if 
$E<C_{\max } . F(X)=0$, otherwise. Crossover operator is designed as follows: Suppose two individuals of a paired group in parent generation $X_{1}=\left(x_{1}^{(1)}, \cdots, x_{n}^{(1)}\right), X_{2}=\left(x_{1}^{(2)}, \cdots, x_{n}^{(2)}\right) . n$ random numbers $\alpha_{i}$ are generated within space interval $(0,1)$. Individuals in child generation after implementing crossover operation are $Y_{1}=\left(y_{1}^{(1)}, \cdots, y_{n}^{(1)}\right)$, $Y_{2}=\left(y_{1}^{(2)}, \cdots, y_{n}^{(2)}\right), y_{i}^{(1)}=\alpha_{i} x_{i}^{(1)}+\left(1-\alpha_{i}\right) x_{i}^{(2)}, y_{i}^{(2)}=\alpha_{i} x_{i}^{(2)}+\left(1-\alpha_{i}\right) x_{i}^{(1)}$. Nonuniformity mutation operator is adopted in this paper. Suppose $k-t h$ component of $X=\left(x_{1}, \cdots, x_{n}\right)$ is selected to implement mutation operation. Individual in child generation after implementing mutation operation is $X^{\prime}=\left(x_{1}, x_{2}, \cdots, x_{k}^{\prime}, \cdots, x_{n}\right)$. Muted gene $x_{k}^{\prime}$ is calculated by the formula: $x_{k}^{\prime}=x_{k}+\Delta\left(t, b_{k}-x_{k}\right)$, if $r n d(2)=0 . x_{k}^{\prime}=x_{k}+\Delta\left(t, x_{k}-a_{k}\right)$, otherwise. Where $\Delta(t, z)=z \cdot\left(1-r^{(1-1 / T)^{\lambda}}\right), \Delta(t, z) \in[0, z]$.

\subsection{Regulation of Crossover and Mutation Probability}

In this paper, fitting density difference of adjacent two generations is used to estimate varying tendency of convergence. The following technique is adopted to regulate crossover and mutation probability.

Suppose set of the $i$-th generation fitting density is: $F_{i}=\left\{F_{i 1}, F_{i 2}, \cdots, F_{i n}\right\}$. Mean of sample $F_{i}$ is $E\left(F_{i}\right)=\frac{1}{n} \sum_{j=1}^{n} F_{i j}$. Standard deviation of sample $F_{i}$ is $S_{F_{i}}^{2}=\frac{1}{n} \sum_{j=1}^{n}\left(F_{i j}-E\left(F_{i}\right)\right)^{2}$. Covariance of sample $F_{i}$ and $F_{j}$ is $S_{F_{i} F_{i}}=\frac{1}{n} \sum_{k=1}^{n}\left(F_{i k}-E\left(F_{i}\right)\right)\left(F_{j k}-E\left(F_{j}\right)\right)$. Correlation coefficient of sample $F_{i}$ and $F_{j}$ is $R_{r_{i} F_{j}}=\frac{S_{l i_{i} F_{j}}}{S_{F_{i}} S_{F_{j}}}$. When number of current evolution generation is less than half of the largest number of evolution generation, crossover probability is regulated. If $R_{F_{i} F_{i}}$ decreases more than 0.1 , crossover probability is increased by 0.1 . Crossover probability is set to initial value otherwise. When number of current evolution generation is more than half of the largest number of evolution generation, mutation probability is regulated. If $R_{F_{i} F_{i}}$ increases more than 0.03 , mutation probability is increased by 0.03 . Mutation probability is set to initial value otherwise. 


\section{EXPERIMENTAL RESULTS AND DISCUSSIONS}

To construct the morphable model, 200 prototypic 3D faces are selected. 24.7 minutes is needed to reconstruct a $3 \mathrm{D}$ face with $2 \mathrm{GHz}$ Pentium 4 processor using our new algorithm (our improved genetic algorithm) while the conventional method (stochastic gradient descent method) needs 33.8 minutes to do the same. Synthesis results are shown in Figure 1. Left column is input image, second column is result of new matching method and last column is result of conventional one.

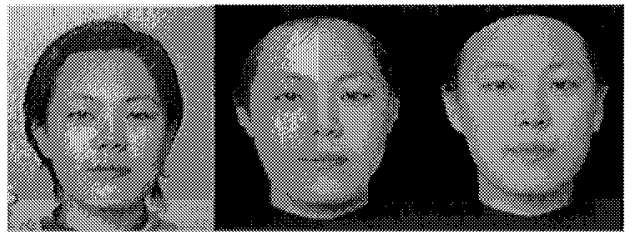

Figure. I Results of reconstructed $3 D$ faces

3D faces reconstructed using two methods are compared with 3D face of same person scanned by the Cyberware. Relative error of corresponding vertexes on two types of 3D face model is defined as the criteria to compare synthesis precision. Error at each vertex is the sum of ratios of coordinate difference to the scale of corresponding coordinate axis along $\mathrm{X}, \mathrm{Y}$ and $\mathrm{Z}$ axis. Figure 2 shows synthesis error decreased by our new algorithm compare to the conventional one.

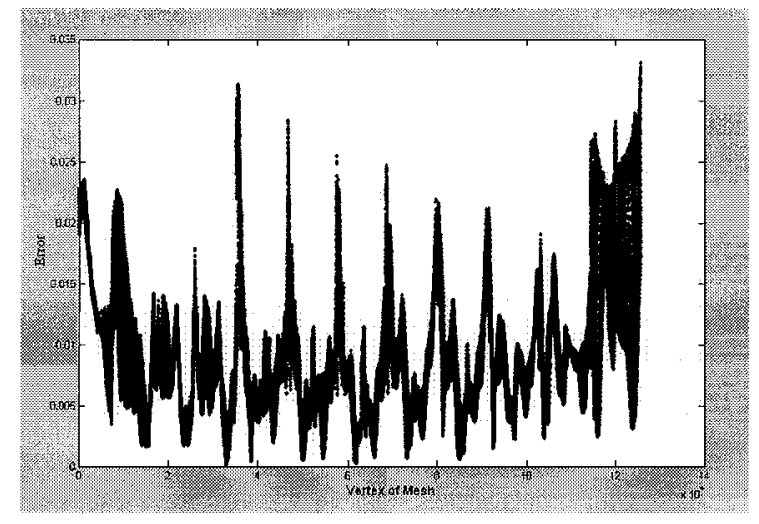

Figure. 2 Decreased error

Experimental results show that our new model matching method based on improved genetic algorithm performs appealing on 3D face synthesis. 
Time consumed by our improved genetic algorithm is less than stochastic gradient descent method under the same conditions. As shown in Figure 1, 9.1 minutes is saved to synthesize a $3 \mathrm{D}$ face using our new model matching method. 3D face synthesis precision is improved by our new model matching method. As shown in Figure 2 maximum synthesis error can be decreased by 3.3 percent

\section{CONCLUSIONS}

In this paper, we propose a novel model matching method based on improved genetic algorithm for 3D face synthesis. Our new method overcomes weaknesses of stochastic gradient descent method. Regulation of crossover and mutation probability during optimization process improves precision and convergence speed of the algorithm. Experimental results show our new matching method based on improved genetic algorithm improves convergence speed, precision and can be applied effectively to solve the model matching problem for 3D face synthesis.

\section{ACKNOWLEDGEMENTS}

This work is supported by the Beijing Educational Committee Project of China (2002KJ001, KM200310005025), the National Natural Science Project of China (60375007).

\section{REFERENCES}

[1] Parke F. I. A Parametric Model of Human Faces. PhD thesis, Salt Lake City: University of Utah, 1974.

[2] Pighin F, Hecker J, et al. Synthesizing realistic facial expressions from photographs. In Proceedings of SIGGRAPH'98, Orlando, Florida: ACM Press, 1998: 75-84.

[3] Lee W S, Thalmann N M. Fast Head Modeling for Animation. Journal Image and Vision Computing, 2000, 18 (4): 355-364.

14] Blanz V, Vetter T. A morphable model for the synthesis of 3D faces. In Proceeding of SIGGRAPH'99, Los Angeles: ACM Press, 1999: 187-194.

[5] Fletcher, R., Practical Methods of Optimization, Vol. 1: Unconstrained Optimization, John Willy and Sons, Chichester, 1980.

[6] Cyberware Laboratory Inc, http://www.cyberware.com

[7] Ozcan E., Onbasioglu E., Genetic algorithms for parallel code optimization. Congress on Evolutionary Computation, 19-23 June, 2004, 2(2): 1375-1381. 